\title{
SOLUBILITY AND DISSOLUTION ENHANCEMENT OF IVACAFTOR TABLETS BY USING SOLID DISPERSION TECHNIQUE OF HOT-MELT EXTRUSION - A DESIGN OF EXPERIMENTAL APPROACH
}

\author{
PURNACHANDRA REDDY GUNTAKA*, SRINIVAS LANKALAPALLI \\ Department of Pharmaceutics, GITAM Institute of Pharmacy, GITAM University, Rushikonda, Visakhapatnam, Andhra Pradesh, India. \\ Email: gpcreddy86@gmail.com
}

Received: 19 October 2018, Revised and Accepted: 22 November 2018

\section{ABSTRACT}

Objective: The objective was to improve the solubility and dissolution of ivacaftor tablets by using solid dispersion (SD) technique.

Methods: Ivacaftor is practically insoluble $(<0.001 \mathrm{mg} / \mathrm{mL})$ over $\mathrm{pH}$ value of 3.0-7.5 due to low solubility, and it shows poor bioavailability after oral administration. Therefore, SDs of Ivacaftor were prepared by SD technique of hot-melt extrusion (HME) by adding different polymers such as Soluplus, Hypromellose $5 \mathrm{cps}$, and Copovidone with surfactants sodium lauryl sulfate, poloxamer, and polysorbate 80 to enhance its solubility.

Results: The analysis of X-ray diffraction and differential scanning calorimetry of Solid dispersion by HME represents the polymorphic conversion of ivacaftor from crystalline structure form to an amorphous structure form. The results show that the formulation of Ivacaftor SDs by HMT has enhanced the solubility and dissolution of Ivacaftor.

Conclusion: In the present study, the SDs of the poorly soluble drug substance Ivacaftor were successfully prepared using HME. The in vitro dissolution test shows a significant increase in dissolution rate of SDs prepared by HME (95\%) in formulation FHM8 compared with plain Ivacaftor (9\%) within $30 \mathrm{~min}$.

Keywords: Ivacaftor, Solid dispersion, Hot-melt extrusion, Soluplus, Copovidone, Hypromellose 5 cps, Sodium lauryl sulfate, Poloxamer and polysorbate 80 .

(c) 2019 The Authors. Published by Innovare Academic Sciences Pvt Ltd. This is an open access article under the CC BY license (http://creativecommons. org/licenses/by/4. 0/) DOI: http://dx.doi.org/10.22159/ajpcr.2019.v12i1.30369

\section{INTRODUCTION}

For absorption of oral formulations, solubility and permeability are act as two major factors. Nowadays, some of the new chemical entities are poor water solubility drugs. Therefore, improving the drug bioavailability is the most tough challenge of solid oral dosage formulations. Solid dispersion is one of the most successful technologies to enhance the solubility of drug. In this approach, drug is converted its polymorphic form by emerged with inert carrier. The conversion of amorphous form drug can easily contact with dissolution media and become more solubility and bioavailability [1-3].

The process of hot melt extrusion (HME) is one of the solid dispersion (SD) technique that efficient mixing of drug with polymer and inert excipient, progressive melting and finally solidified. The drugpolymer ratio, quantity of surfactant, selection of polymer, extrusion temperature, and screw rotation speed are important parameters; it can affect quality and solubility of drug product. For example, the screw rotation speed may impact the proper conversion of polymorphic form and mixing of polymer and surfactant $[4,5]$.

Fig 1, represents Chemical structure of Ivacaftor is a cystic fibrosis transmembrane conductance regulator potentiator indicated for the treatment of cystic fibrosis. Ivacaftor is a white to off-white crystalline powder. The crystalline form of the drug substances is practically insoluble in aqueous media $(<0.001 \mathrm{mg} / \mathrm{ml})$. To increase the solubility of Ivacaftor, HME technology was selected. Therefore, the selection of polymer carrier able to conversion of polymorphic form of ivacaftor, along with effects keeping ivacaftor in amorphous state $[6,7]$.

The present study was carried out to develop Ivacaftor SD by HME (Fig. 2). Technology using three different polymers and three different surfactants of enhancing drug solubility. By different prototype formulations select one optimize formulation based on dissolution profile and perform factorial design.

\section{MATERIALS AND METHODS}

Materials

Ivacaftor drug substance was gift sample of Aurobindo pharma Ltd., Hyderabad, India. Cellulose microcrystalline (Avicel pH 102) was gifted by FMC Biopolymer, USA. Hypromellose 5 cps was gift sample of Dow Chemical, USA. Aerosil 200 (colloidal silicon dioxide) was gifted by Evonik, Germany. Polyvinyl acetate-polyvinyl caprolactam-polyethylene glycol graft copolymer, grade Soluplus and Copovidone, sodium lauryl sulfate, and poloxamer were gift sample of BASF, and polysorbate 80 was gifted by Seppic. Croscarmellose sodium was gifted by DFE Pharma, Germany, and magnesium stearate was given by Peter Greven, the Netherlands.

\section{HME}

Preparation of Ivacaftor SDs by HME

Ivacaftor SD was prepared by different carriers such as Copovidone, Soluplus, and Hypromellose 5 cps along with surfactants such as sodium lauryl sulfate, poloxamer, and polysorbate 80. HME Pharma 24 - Thermo Fisher twin screw model was used for the preparation of SDs using feed rate of $1-1.3 \mathrm{Kg} / \mathrm{h}$, torque: $4 \mathrm{Barr}$, and 10 different temperature zones from $20^{\circ} \pm 2^{\circ} \mathrm{C}$ to $210^{\circ} \pm 2^{\circ} \mathrm{C}$ with chillers zone maintain at temperature $2-5^{\circ} \mathrm{C}$ (where melt was converted into flake pieces).

\section{Manufacturing process}

- Step 1: Ivacaftor was taken with Copovidone (one set of trials), Hypromellose $5 \mathrm{cps}$ (second set of trials), and Soluplus (third set of trials) along with surfactants such as sodium lauryl sulfate, poloxamer. 
and polysorbate 80 which were sifted through \#40 mesh and mixed well using poly bag for $10 \mathrm{~min}$. Polysorbate 80 is available in liquid state so dilute with water as $10 \%$ solids and coating into plain Ivacaftor API by using FBP. The above material was hot-melt extruded using above mentioned at different temperature zones (Table 1). Table 2, represents composition of Ivacaftor by HME. The extrudes were transparent in FHM2, FHM3, FHM5, FHM6, FHM8, and FHM9. Remaining extrudes were opaque in nature. The extrudes crushed into mortar and pestle. The powder was granular in nature and sifted through \#30 mesh.

- $\quad$ Step 2: The extrudes of step no 1, cellulose microcrystalline (Avicel pH 102), croscarmellose sodium (Ac-Di-Sol), and colloidal silicon dioxide (Aerosil 200) were sifted through \#30 mesh and mixed well using poly bag for $10 \mathrm{~min}$.

- $\quad$ Step 3: Magnesium stearate sifted through \#40 mesh and added to step no 2 mixed in poly bag for 5 min manually.

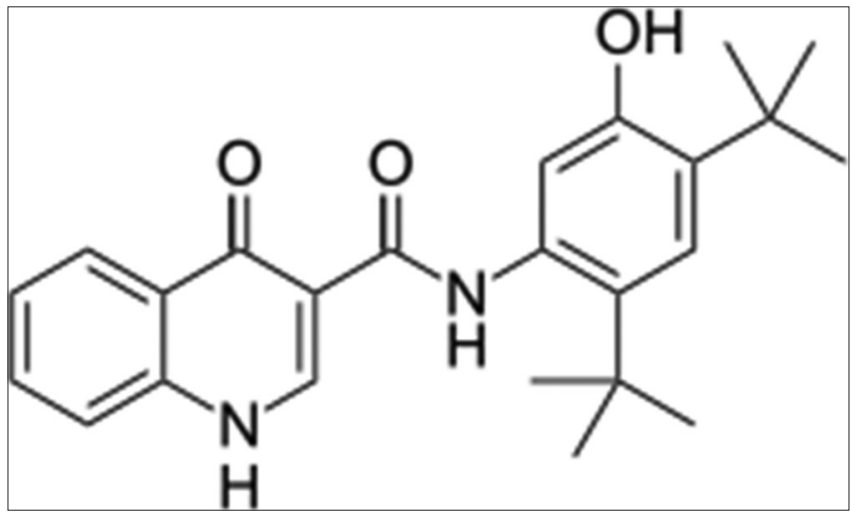

Fig. 1: Chemical structure of Ivacaftor

Table 1: Temperatures to be monitored during HME process

\begin{tabular}{ll}
\hline Name of zones & Temperature \\
\hline $\begin{array}{l}\text { Barrel conveying } \\
\text { unit/zone I }\end{array}$ & $20^{\circ} \mathrm{C} \pm 2^{\circ} \mathrm{C}$ \\
Zone II & \\
Zone III & $20^{\circ} \mathrm{C} \pm 2^{\circ} \mathrm{C}$ \\
Zone IV & $80^{\circ} \mathrm{C} \pm 2^{\circ} \mathrm{C}$ \\
Zone V & $120^{\circ} \mathrm{C} \pm 2^{\circ} \mathrm{C}$ \\
Zone VI & $180^{\circ} \mathrm{C} \pm 2^{\circ} \mathrm{C}$ \\
Zone VII & $210^{\circ} \mathrm{C} \pm 2^{\circ} \mathrm{C}$ \\
Zone VIII & $210^{\circ} \mathrm{C} \pm 2^{\circ} \mathrm{C}$ \\
Zone IX & $210^{\circ} \mathrm{C} \pm 2^{\circ} \mathrm{C}$ \\
Zone X & $80^{\circ} \mathrm{C} \pm 2^{\circ} \mathrm{C}$ \\
Die zone & $50^{\circ} \mathrm{C} \pm 2^{\circ} \mathrm{C}$ \\
Chillers/cooling & $20^{\circ} \mathrm{C} \pm 2^{\circ} \mathrm{C}$ \\
zone & Maintained at temperature $2-5^{\circ} \mathrm{C}$ \\
\hline
\end{tabular}

- $\quad$ Step 4: The lubricated blend of step no 3 was compressed using $12.00 \mathrm{~mm}$ round-shaped punches.

\section{Evaluation of Ivacaftor SDs}

Solubility studies of Ivacaftor SDs

Solubility measurements of Ivacaftor were performed with solvent shaken for the $12 \mathrm{~h}$ at room temperature. Subsequently, the suspensions were filtered through a Whatman filter paper. A filtered solution of Ivacaftor was analyzed using UV $255 \mathrm{~nm}$.

\section{Drug content}

Solid dispersions equivalent to $150 \mathrm{mg}$ of Ivacaftor were weighed accurately and dissolved in $100 \mathrm{ml}$ of $0.1 \%$ trifluoroacetic acid: acetonitrile (20:80 ratio). The solution was filtered and diluted with the suitable amount, and drug content was analyzed at $\ddot{e}_{\text {max }} 255 \mathrm{~nm}$ against blank using UV spectrometer [8]. The actual drug content was calculated using the following equation:

$\%$ Drug content $=\frac{\text { Actual amount of drug in solid dispersion }}{\text { Theoretical amount of drug in solid dispersion }} \times 100$

\section{In vitro drug release studies}

The in vitro drug release profile for each SD as well as plain drug was performed using USP type 2 dissolution apparatus. The sample equivalent to $150 \mathrm{mg}$ of Ivacaftor was added and the conditions maintained are shown in Table 3.

The samples were drawn at specified time intervals, and the obtained samples were analyzed using UV-visible spectrophotometer at $255 \mathrm{~nm}$. The cumulative percentage release was calculated [9].

\section{RESULTS AND DISCUSSION}

Fourier-transform infrared (FT-IR) spectrometry studies

FT-IR spectrum majorly was used to determine if any of interaction between the drug and excipient used. The prominent peaks of Ivacaftor were observed (Fig. 3) the region: $3332 \mathrm{~cm}^{-1}$ due to $>\mathrm{N}-\mathrm{H}(\mathrm{N}-\mathrm{H}$ stretching), $2957 \mathrm{~cm}^{-1}$ due to $-\mathrm{O}-\mathrm{H}$ (-OH stretching), and $1647 \mathrm{~cm}^{-1}$ due to $-\mathrm{C}=\mathrm{O}$ (stretch). The optimized formulation FSD8 (Fig. 4) displayed the characteristic peaks at wave numbers nearer to that of plain Ivacaftor (Fig. 3). Overall, there was no alteration in the characteristic peaks of the optimized formulation, suggesting that there was no interaction between the drug and polymers.

\section{Differential scanning calorimetry (DSC)}

The DSC thermogram of plain Ivacaftor is shown in Fig. 5, sharp peak of endothermic at $205^{\circ} \mathrm{C}$ melting point, indicating that the drug was crystalline. The absence of peaks in the SD of formulation FHM8 (Ivacaftor: Soluplus (1:1) with poloxamer) indicates that the drug was converted in amorphous form [10].

Table 2: Composition of Ivacaftor SDs by HME

\begin{tabular}{|c|c|c|c|c|c|c|c|c|c|c|}
\hline S. No & Ingredients (Units) & FHM1 & FHM2 & FHM3 & FHM4 & FHM5 & FHM6 & FHM7 & FHM8 & FHM9 \\
\hline 1. & Ivacaftor (mg) & 150.0 & 150.0 & 150.0 & 150.0 & 150.0 & 150.0 & 150.0 & 150.0 & 150.0 \\
\hline 2. & Copovidone (mg) & 150.0 & 150.0 & 150.0 & - & - & - & - & - & - \\
\hline 3. & Hypromellose 5 cps (mg) & - & - & - & 150.0 & 150.0 & 150.0 & - & - & - \\
\hline 4. & Soluplus (mg) & - & - & - & - & - & - & 150.0 & 150.0 & 150.0 \\
\hline 5. & Sodium lauryl sulfate (mg) & 15.0 & - & - & 15.0 & - & - & 15.0 & - & - \\
\hline 6. & Poloxamer (mg) & - & 15.0 & - & - & 15.0 & - & - & 15.0 & - \\
\hline 7. & Polysorbate 80 (mg) & - & - & 15.0 & - & - & 15.0 & - & - & 15.0 \\
\hline 8. & Microcrystalline cellulose (Avicel pH 102) (mg) & 214.5 & 214.5 & 214.5 & 214.5 & 214.5 & 214.5 & 214.5 & 214.5 & 214.5 \\
\hline 9. & Croscarmellose sodium (Ac-Di-Sol) (mg) & 12.5 & 12.5 & 12.5 & 12.5 & 12.5 & 12.5 & 12.5 & 12.5 & 12.5 \\
\hline 10. & Colloidal silicon dioxide (Aerosil 200) (mg) & 3.0 & 3.0 & 3.0 & 3.0 & 3.0 & 3.0 & 3.0 & 3.0 & 3.0 \\
\hline \multirow[t]{2}{*}{11.} & Magnesium stearate (mg) & 5.0 & 5.0 & 5.0 & 5.0 & 5.0 & 5.0 & 5.0 & 5.0 & 5.0 \\
\hline & Total tablet weight (mg) & 550.0 & 550.0 & 550.0 & 550.0 & 550.0 & 550.0 & 550.0 & 550.0 & 550.0 \\
\hline
\end{tabular}

HME: Hot-melt extrusion, SD: Solid dispersions 


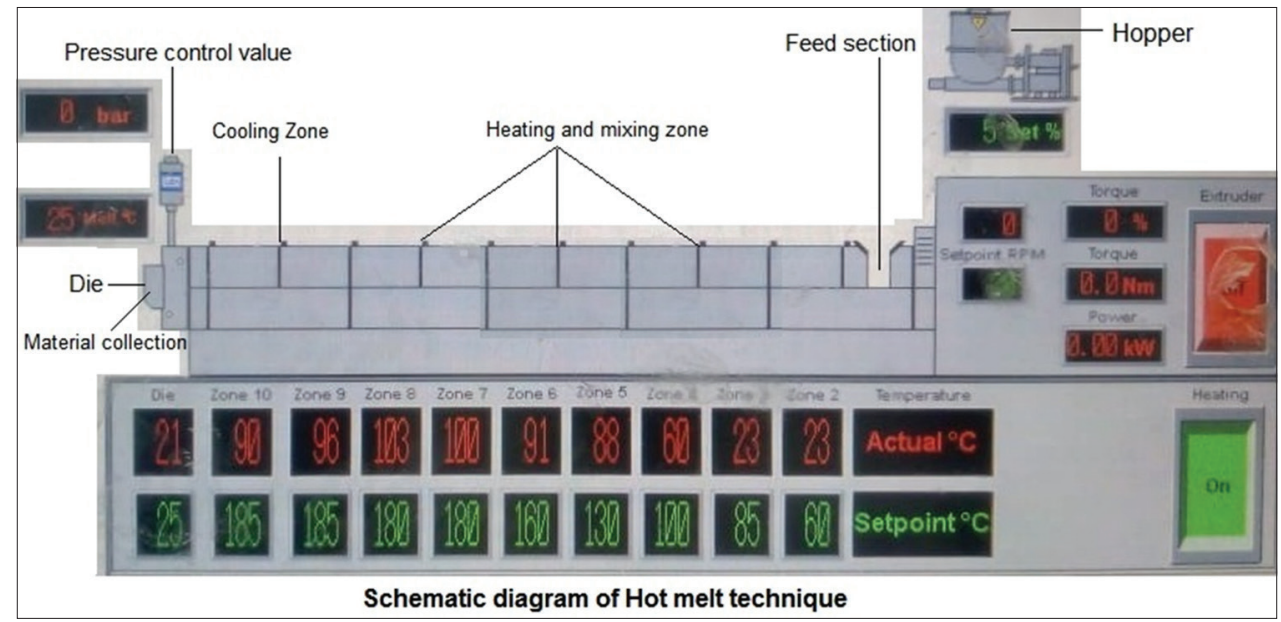

Fig. 2: Schematic diagram of hot-melt extrusion

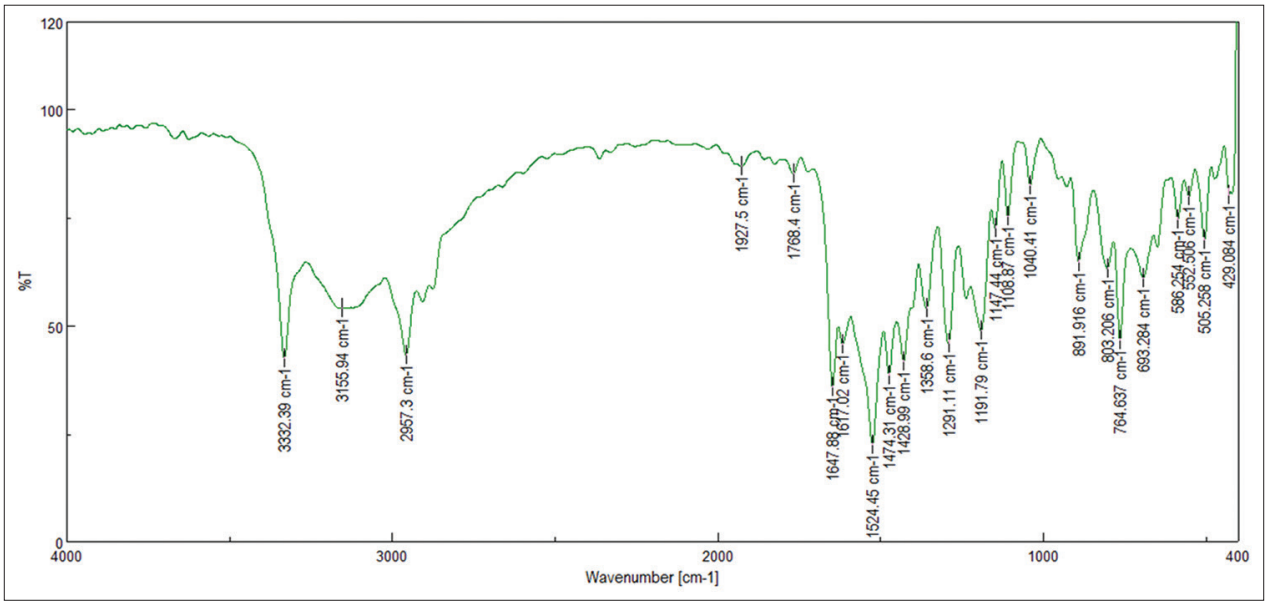

Fig. 3: Fourier-transform infrared spectra of plain drug

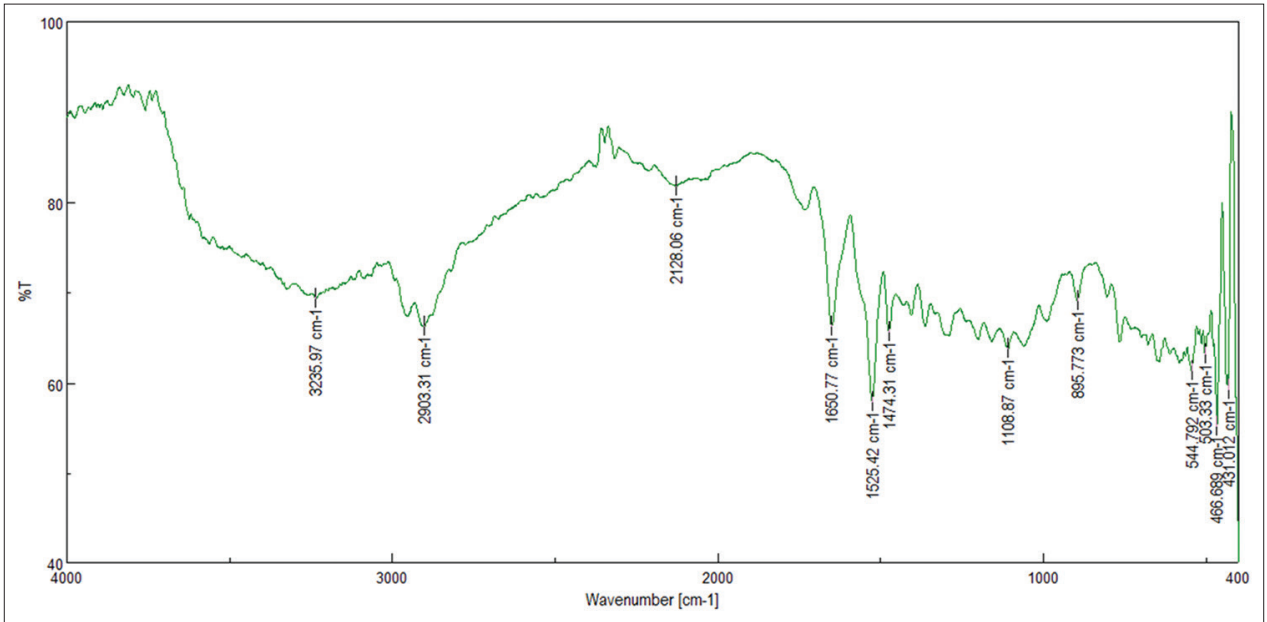

Fig. 4: Fourier-transform infrared spectra of formulation FSD8 solid dispersion

\section{X-ray diffraction (XRD) analysis}

TheXRD of Ivacaftor observed that multiple sharp peaks (Fig. 6) indicates that the drug was in crystalline nature. SD optimized formulation FHM8 (Ivacaftor: Soluplus (1:1) with poloxamer) when exposed to X-ray beam observed no crystalline endothermic peaks and characteristic intensities of Ivacaftor (Fig. 7). This indicates that complete conversion of crystalline Ivacaftor into amorphous polymorphic state during hot melt extrusion process. From the XRD studies, it is clearly confirmed that HME of batch no FHM8 drug substance converted into amorphous form [11].

Scanning electron microscopy (SEM)

HMEs of FHM8 performed surface micrographs, and plain Ivacaftor was determined into SEM technique. The SEM micrograph of plain Ivacaftor 


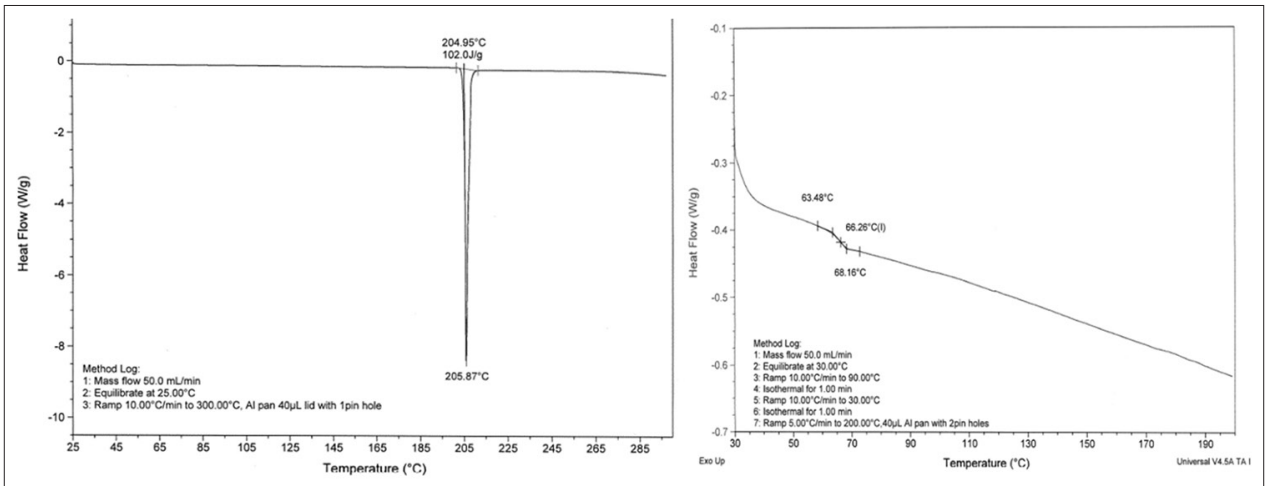

Fig. 5: Differential scanning calorimetry thermograms of plain drug and optimized formulation FHM8

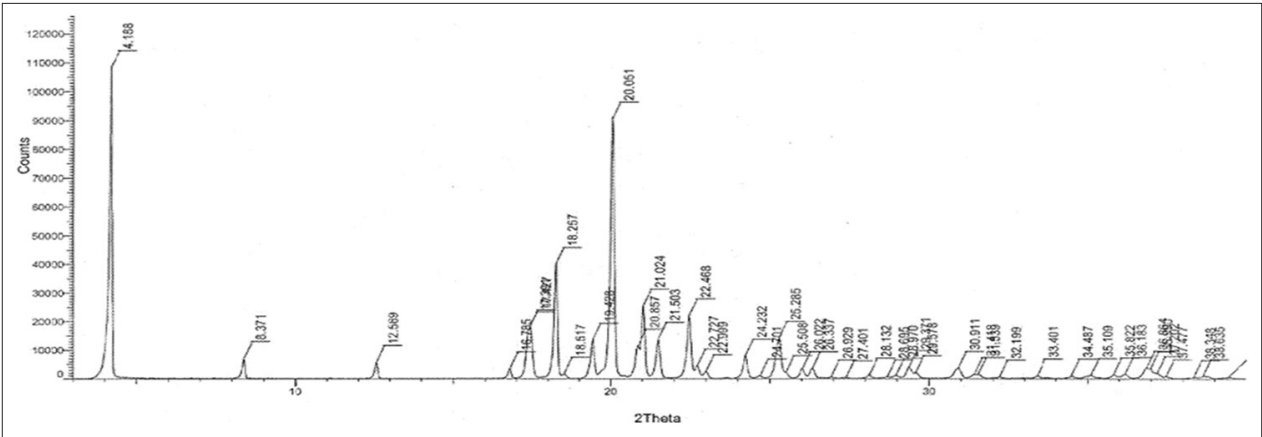

Fig. 6: Powder X-ray diffraction patterns of Ivacaftor plain drug

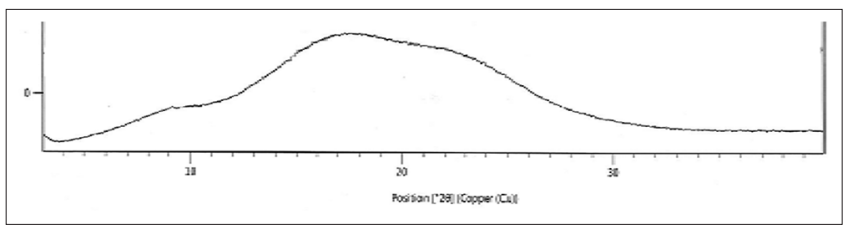

Fig. 7: Powder X-ray diffraction patterns of the optimized formulation of FHM8

Table 3: In vitro dissolution studies of test parameters

\begin{tabular}{ll}
\hline Instrument & $\begin{array}{l}\text { Electrolab - USP type II dissolution test } \\
\text { apparatus } \\
\text { pH } 6.8 \text { sodium phosphate buffer with } 0.7 \%\end{array}$ \\
Dissolution & $\begin{array}{l}\text { sodium dodecyl sulfate } \\
\text { medium }\end{array}$ \\
Apparatus & $37 \pm 0.5^{\circ} \mathrm{C}$ \\
Temperature & 65 \\
RPM & $900 \mathrm{ml}$ \\
Volume of medium & $5,10,15,20$, and 30 min \\
Sampling intervals & $10 \mathrm{ml}$ withdrawn and replaced with $10 \mathrm{ml}$ of \\
Sample volume & dissolution medium \\
\hline
\end{tabular}

Table 4: Solubility in aqueous media and drug content of SDs prepared by HME method

\begin{tabular}{llll}
\hline S. No. & Formulation & Solubility $(\mathrm{mg} / \mathrm{ml})$ & \% Drug content \\
\hline 1 & Plain drug & 0.001 & - \\
2 & FHM8 & 0.25 & $98.5 \%$ \\
\hline
\end{tabular}

HME: Hot-melt extrusion, SD: Solid dispersions

(Fig. 8) was observed crystalline drug agglomerates with ordered shape and size (Fig. 8). The surface characteristics extrude of optimized formulation FHM8 [12,13].

\section{Evaluation parameters}

Solubility studies of Ivacaftor SDs

Nine formulations of the SDs were prepared using HME technique with respective polymer. After preparation of SDs using HME process, the resulting extruded mixture was analyzed for solubility of drug and was compared with plain drug itself (Table 4). The formulation of (Ivacaftor: soluplus (1:1) with poloxamer) FHM8 represents the solubility enhancement as compared to plain drug (plain drug solubility is $0.001 \mathrm{mg} / \mathrm{ml}$ ) [14,15].

\section{In vitro dissolution studies}

The obtained drug release data for formulations FHM1 to FHM9 are shows in Fig 9. Table 6 shows the cumulative percentage drug released for all formulations. Cumulative percentage of drug released after 30 min was $72 \%, 77 \%, 78 \%, 80 \%, 85 \%, 82 \%, 89 \%, 95 \%$, and 93\% for FHM1-FHM9, respectively, and was $9 \%$ in $30 \mathrm{~min}$ for plain drug. In vitro studies reveal that there is a marked increase in the dissolution rate of Ivacaftor from all the SDs when compared to plain drug itself. From the in vitro drug release profiles, formulation FHM8 containing Ivacaftor: Soluplus (1:1) with surfactant poloxamer was best formulation which shows high dissolution rate, i.e., 95.0\% compared with other formulations. This may attributed to increase the conversion of drug to amorphous (Table 5).

The dissolution profiles of Ivacaftor SDs prepared by HME (FHM8) shown that the \% drug release was more compared with all nine formulations. The SD formulations by FHM8 shown highest drug release, i.e., $95.0 \%$, respectively, after $30 \mathrm{~min}$, where plain drug release was only $9 \%$.

\section{Statistical analysis}

Based on the preliminary feasibility study, a design of experiments (DOE) with full factorial design (Table 7) was performed to optimize Soluplus and poloxamer concentrations used in the formulation. Percentage of drug release in $30 \mathrm{~min}$ was identified as a critical 
Table 5: Physicochemical characteristics of Ivacaftor SD tablets

\begin{tabular}{|c|c|c|c|c|c|}
\hline Batch number & Weight of tablet (mg) & Thickness (mm) & Friability test $(<1 \%)$ & Hardness (KP) & Disintegration (Sec) \\
\hline FHM1 & $550 \pm 4$ & $6.6 \pm 0.2$ & 0.12 & $8 \pm 1$ & 55 \\
\hline FHM2 & $550 \pm 3$ & $6.5 \pm 0.2$ & 0.08 & $8 \pm 2$ & 48 \\
\hline FHM3 & $550 \pm 3$ & $6.5 \pm 0.2$ & 0.06 & $8 \pm 1$ & 40 \\
\hline FHM4 & $550 \pm 4$ & $6.6 \pm 0.1$ & 0.13 & $8 \pm 2$ & 52 \\
\hline FHM5 & $550 \pm 4$ & $6.5 \pm 0.1$ & 0.14 & $7 \pm 2$ & 49 \\
\hline FHM6 & $550 \pm 4$ & $6.5 \pm 0.2$ & 0.09 & $8 \pm 1$ & 48 \\
\hline FHM7 & $550 \pm 3$ & $6.6 \pm 0.2$ & 0.10 & $7 \pm 1$ & 45 \\
\hline FHM9 & $550 \pm 3$ & $6.5 \pm 0.1$ & 0.09 & $8 \pm 1$ & 40 \\
\hline
\end{tabular}

SD: Solid dispersions

Table 6: In vitro dissolution profile of plain drug and different formulations of Ivacaftor SDs (FHM1-FHM9)

\begin{tabular}{|c|c|c|c|c|c|c|c|c|c|c|}
\hline \multirow[t]{2}{*}{ Time (min) } & \multicolumn{10}{|c|}{ Cumulative $\%$ drug release } \\
\hline & Plain drug & FHM1 & FHM2 & FHM3 & FHM4 & FHM5 & FHM6 & FHM7 & FHM8 & FHM9 \\
\hline 0 & 0 & 0 & 0 & 0 & 0 & 0 & 0 & 0 & 0 & 0 \\
\hline 10 & 8 & 51 & 56 & 55 & 61 & 63 & 63 & 62 & 72 & 68 \\
\hline 15 & 8 & 65 & 69 & 68 & 75 & 79 & 78 & 79 & 92 & 86 \\
\hline 20 & 9 & 69 & 75 & 75 & 78 & 83 & 80 & 85 & 94 & 91 \\
\hline 30 & 9 & 72 & 77 & 78 & 80 & 85 & 82 & 89 & 95 & 93 \\
\hline
\end{tabular}

SDs: Solid dispersions

Table 7: Design of the $2^{2}$ full factorial DOE design to study

\begin{tabular}{llll}
\hline Factors: Formulation & \multicolumn{2}{l}{ Levels } & \\
\cline { 2 - 4 } variables (mg) & $\mathbf{- 1}$ & $\mathbf{0}$ & $\mathbf{1}$ \\
\hline A: Soluplus & 75 & 150 & 225 \\
B: Poloxamer & 7.5 & 15 & 22.5 \\
Responses (min) & Goal & Acceptable ranges \\
Y1 & & & \\
Dissolution time & Minimize & Not $<80 \%$ Q in 30 min \\
\hline
\end{tabular}
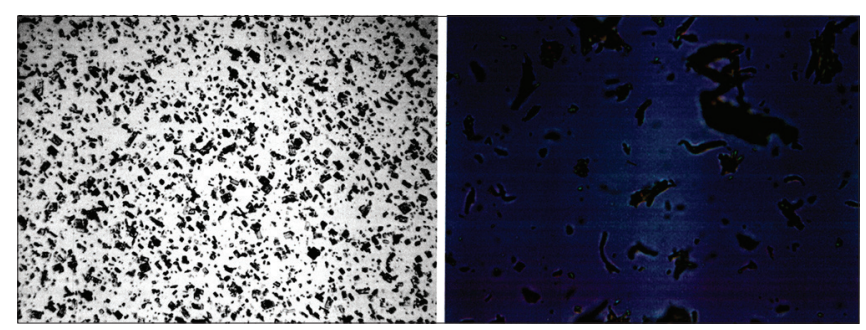

Fig. 8: Scanning electron microscopy images of Ivacaftor plain drug and extrudes of optimized formulation FHM8

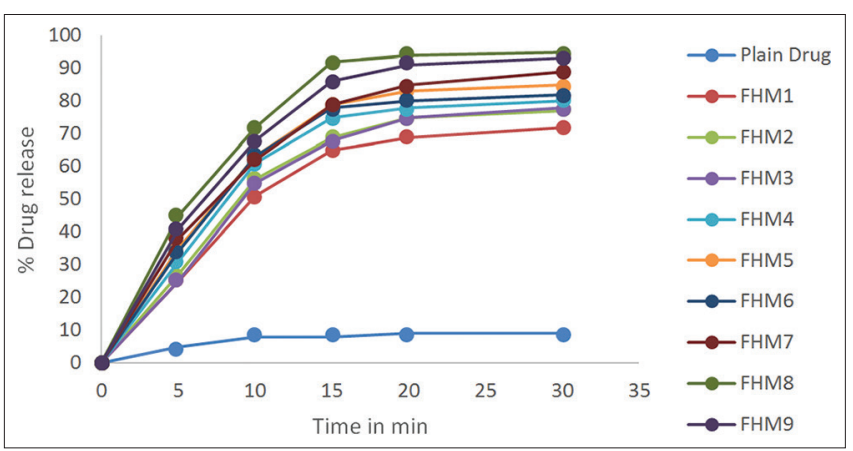

Fig. 9: In vitro dissolution profiles of plain drug and hot-melt extrusion of ivacaftor tablets

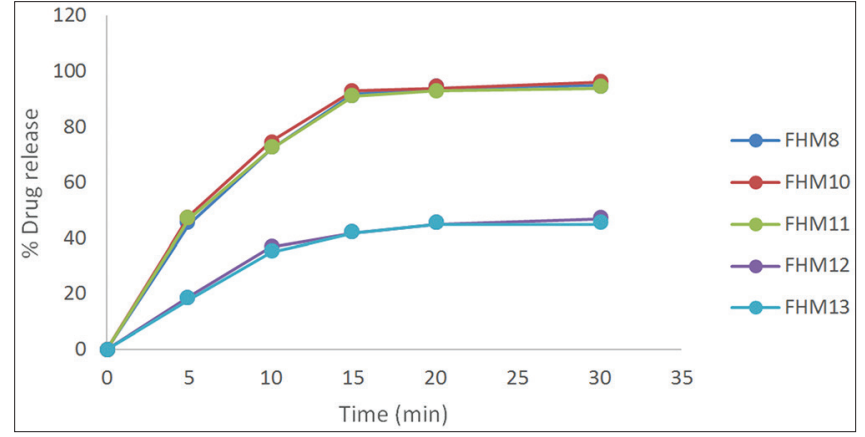

Fig. 10: In vitro dissolution profiles of plain drug and hot-melt extrusion of Ivacaftor tablets

quality attribute of the formulation composition and the ranges for the responses were based on the dissolution of the formulations, and it summarizes the study design and acceptance criteria. Hence, the drug release at $30 \mathrm{~min}$ using USP apparatus II (Paddle) at $65 \mathrm{rpm}$ in pH 6.8 sodium phosphate buffer with $0.7 \%$ sodium dodecyl sulfate, $900 \mathrm{~mL}$, was also evaluated.

A constant tablet weight of $550.00 \mathrm{mg}$ was used by compensating the quantity with the diluent (Microcrystalline cellulose [Avicel pH 102]) to achieve the target weight. The goal of formulation development was to select the optimize Soluplus and poloxamer concentrations and to understand if there was any interaction within the variables. This study also sought to establish the robustness of the proposed formulation. Initially, $2^{2}$ full factorial DOE with one center points was studied, and from the results of the formulation trails using Design-Expert ${ }^{\circledR} 11$ Software, the table no 8 , summarizes the factors as soluplus, poloxamer and responses as dissolution in 30 min studied for subjected to dissolution testing.

The experimental results for dissolution $\left(\mathrm{Y}_{1}\right)$ are presented in Table 8.

Fig 10, represents the \% drug release of optimum batch (FHM8) and DOE trial batches. Using different concentrations of Soluplus shows major effect of dissolution profile. By using soluplus concentration 75 $\mathrm{mg}$ shows the significant effect on dissolution profile and using 150 $\mathrm{mg}, 225 \mathrm{mg}$ represents no significant effect on dissolution. There is 


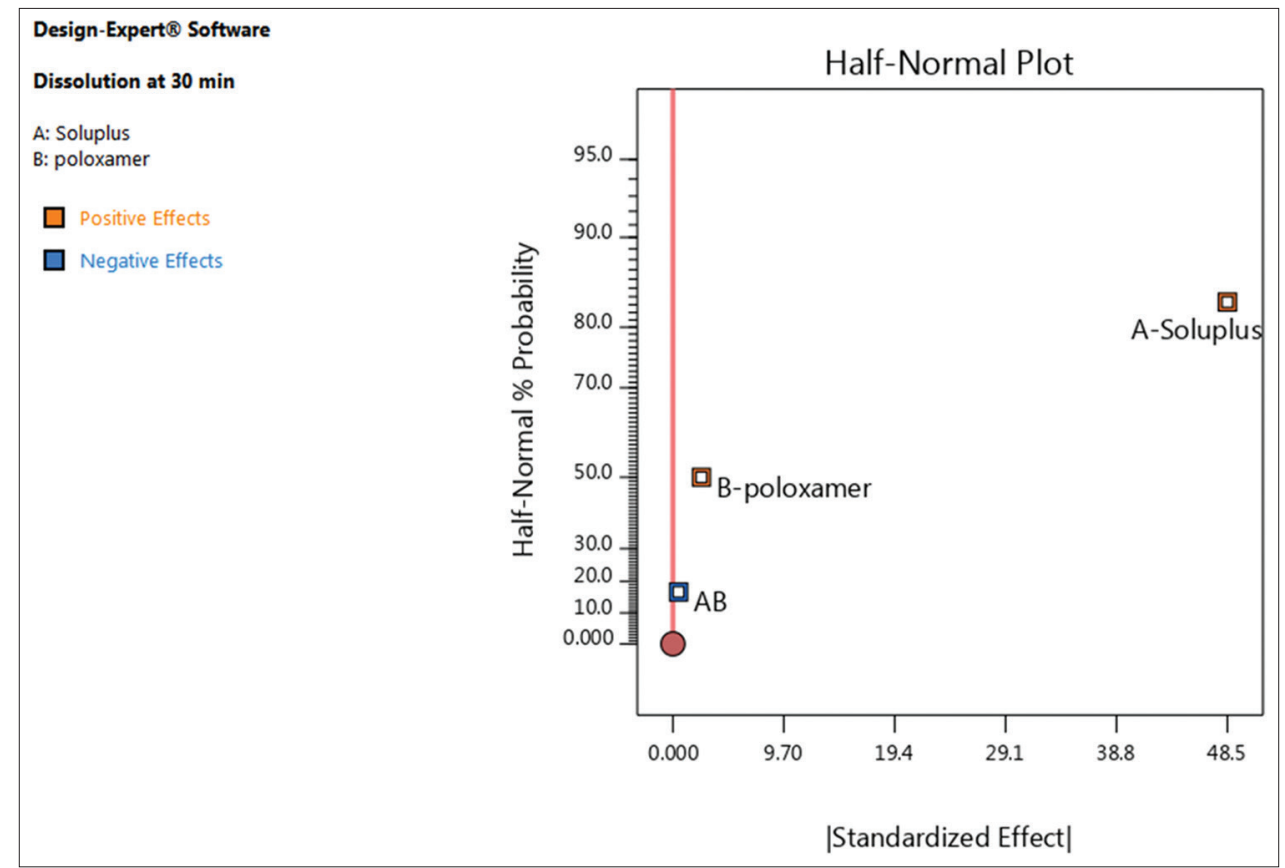

Fig. 11: Half-normal plot

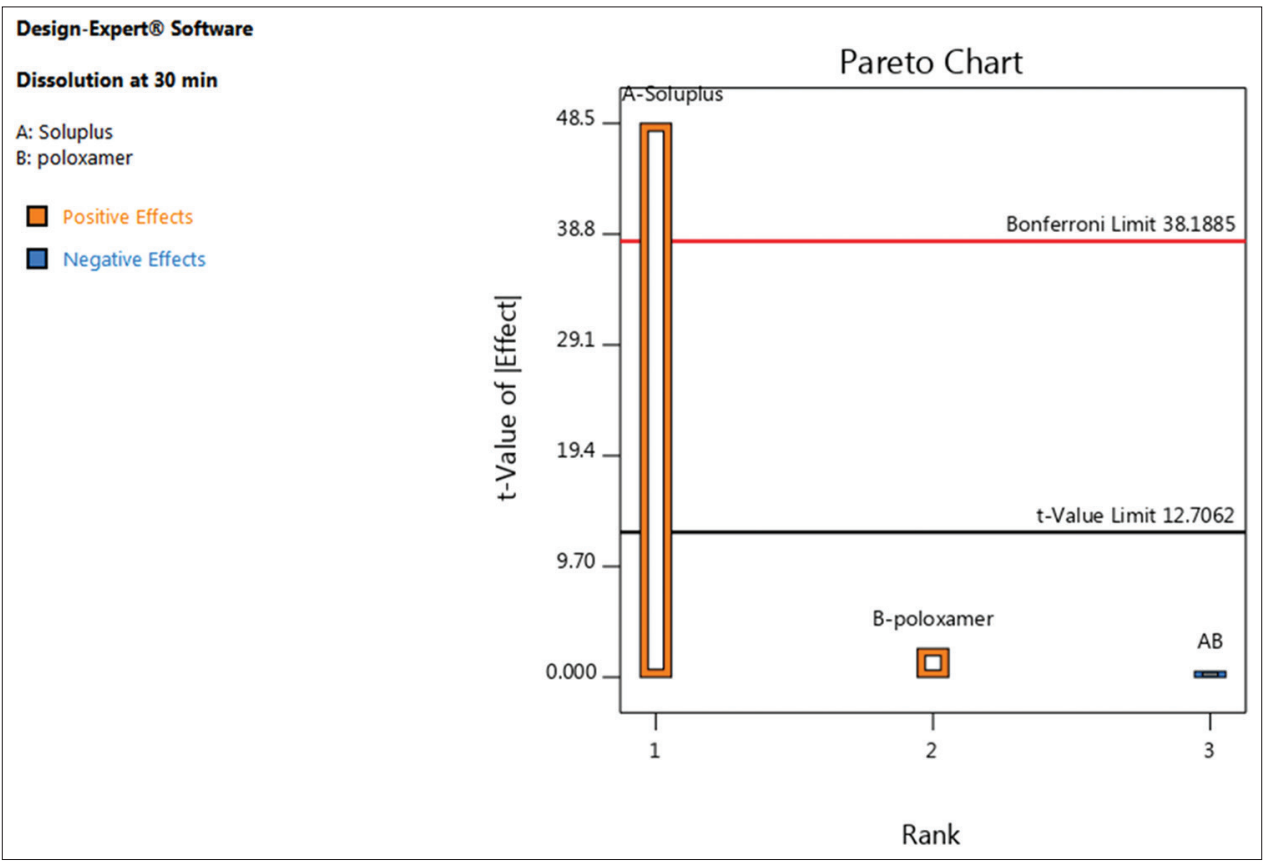

Fig. 12: Pareto chart

Table 8: Experimental results for dissolution $\left(\mathrm{Y}_{1}\right)$

\begin{tabular}{lllll}
\hline S.No & \multicolumn{2}{l}{ Factors : Formulation Variables } & & Responses \\
\cline { 2 - 3 } \cline { 3 - 4 } & Batch no & A: Soluplus (mg) & B: Poloxamer (mg) & \\
\hline 1 & FHM10 & 225 & 22.5 & 96 \\
2 & FHM11 & 225 & 7.5 & 94 \\
3 & FHM8 & 150 & 15 & 95 \\
4 & FHM12 & 75 & 22.5 & 47 \\
5 & FHM13 & 75 & 7.5 & 45 \\
\hline
\end{tabular}

no significant effect by using poloxamer at different concentrations on dissolution was observed. Poloxamer used in different concentrations from 7.5 to 22.5 shows no significant effect of formulation. So there is no much effect on dissolution by using poloxamer. (Table 9)
A two-factor experimental design represents p-value that shows a significant effect of the formulation. $\mathrm{p}<0.05$ shows a significant effect and $p>0.05$ represents the non-significant effect. Data given in Table 10 demonstrate that $\mathrm{p}=0.0103$ which is $<0.05$ the selected model shows a 


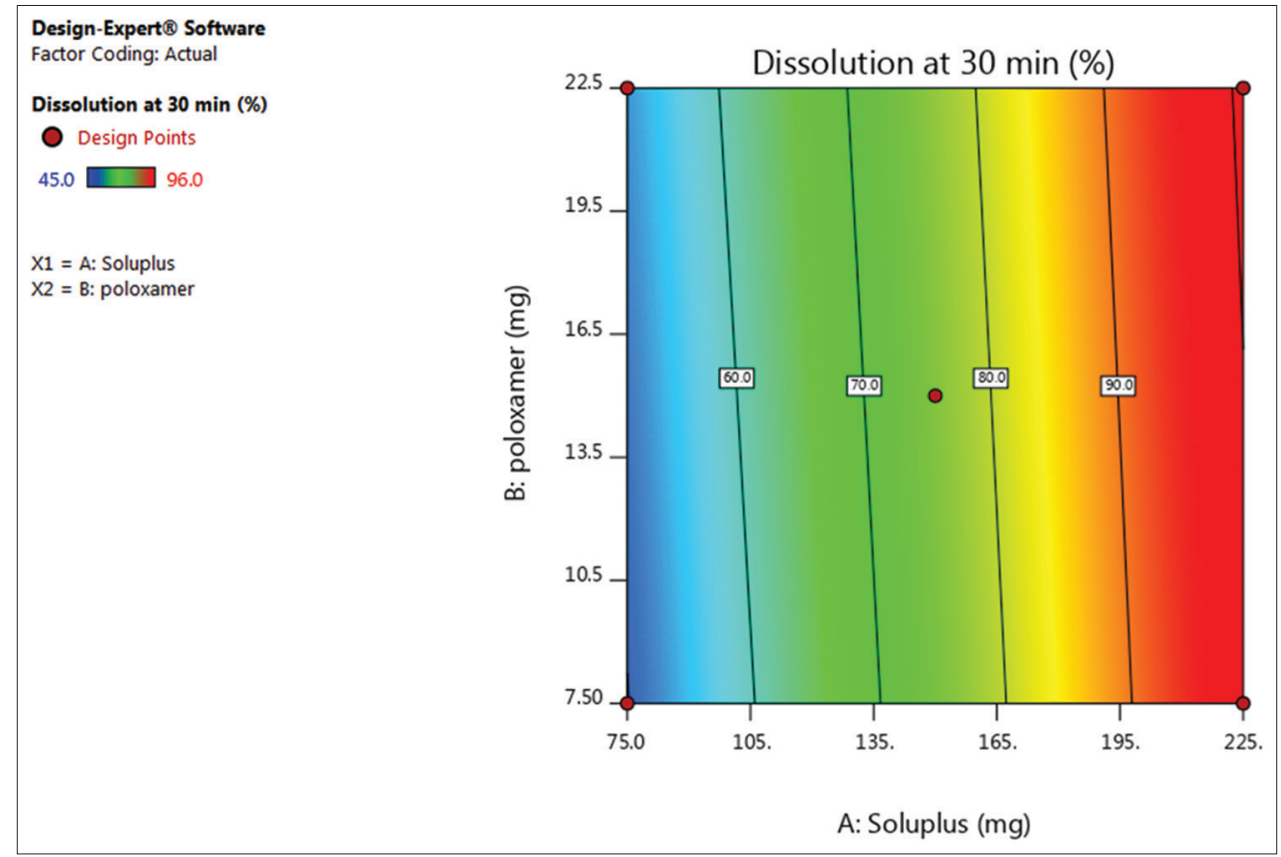

Fig. 13: Contour model plot

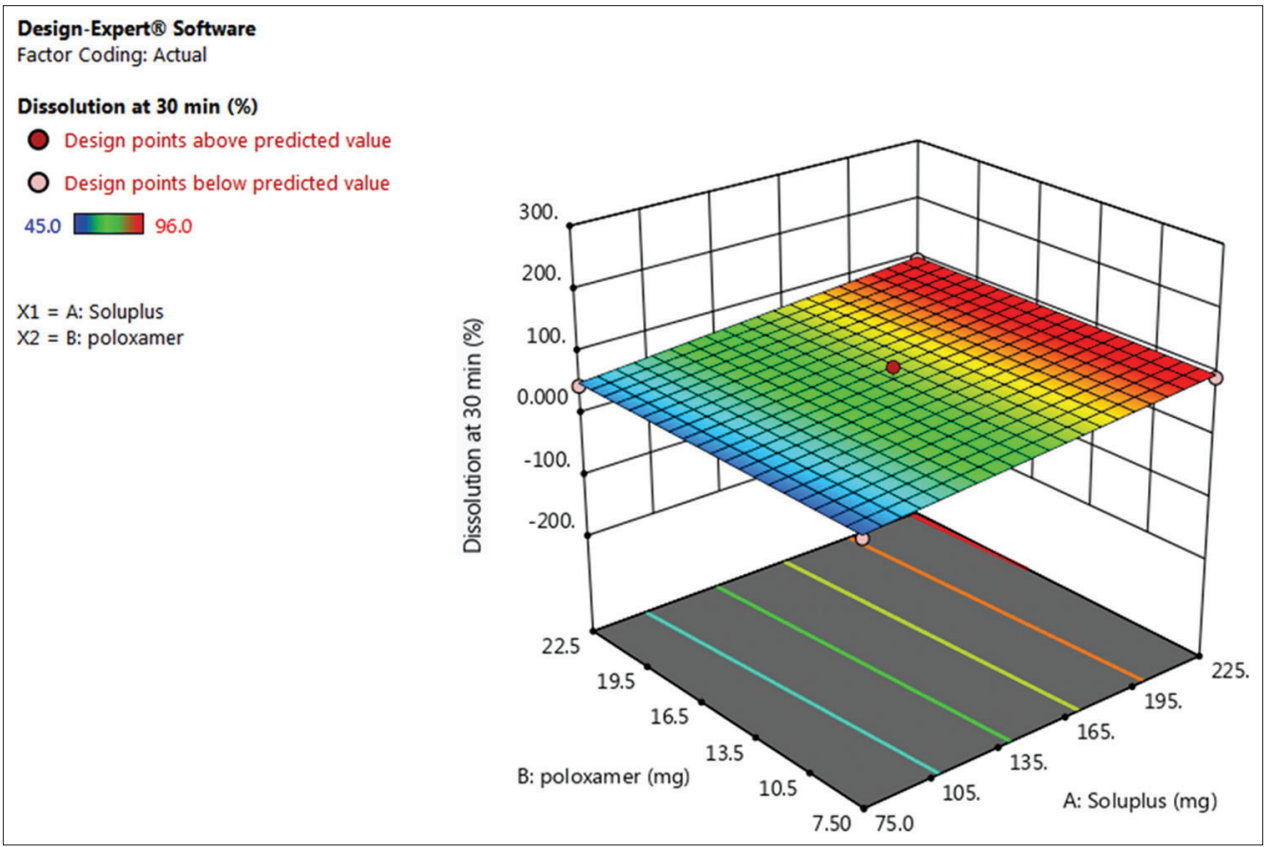

Fig. 14: Three-dimensional response surface plots

Table 9: Dissolution profiles of the formulations

\begin{tabular}{lcllll}
\hline Time (min) & FHM8 & FHM10 & FHM11 & FHM12 & FHM13 \\
\hline \multicolumn{2}{l}{ Dissolution profile } & & & & \\
\hline 5 & 45 & 48 & 47 & 19 & 18 \\
10 & 72 & 75 & 72 & 37 & 35 \\
15 & 92 & 93 & 91 & 42 & 42 \\
20 & 94 & 94 & 93 & 45 & 45 \\
30 & 95 & 96 & 94 & 47 & 45 \\
\hline
\end{tabular}

significant effect. For Soluplus, $\mathrm{p}=0.00656$ shows the significant effect by changing the concentration of Soluplus. For poloxamer $\mathrm{p}=0.126$ which represents not significant effect by using different poloxamer concentrations.

From the above half normal plot, the formulation variables on dissolution in $30 \mathrm{~min}$ shows that significant effect by using different concentrations of soluplus and poloxamer (Fig 12). Soluplus shows the longest effect in halfnormal plot and Pareto chart (Fig 11). Dissolution time in the formulation of the range studied is within the proposed specification limit (NLT 80\% drug release in $30 \mathrm{~min}$ ). Hence, in the present formulation, $150.0 \mathrm{mg}$ per tablet of Soluplus and $15 \mathrm{mg}$ poloxamer per tablet were selected for the finalized formulation.

Two-dimensional contour plots and three-dimensional response surface plots for variables are shown in Figs 13 and 14, respectively. 
Table 10: ANOVA for selected factorial model, response 1: Dissolution

\begin{tabular}{|c|c|c|c|c|c|c|}
\hline Source & Sum of squares & df & Mean square & F-value & p-value & Significant \\
\hline Model & $2.36 \mathrm{E}+03$ & 2 & $1.18 \mathrm{E}+03$ & $4.72 \mathrm{E}+03$ & 0.0103 & \\
\hline A-Soluplus & $2.35 \mathrm{E}+03$ & 1 & $2.35 E+03$ & $9.41 \mathrm{E}+03$ & 0.00656 & \\
\hline B-poloxamer & 6.25 & 1 & 6.25 & 25.0 & 0.126 & \\
\hline Curvature & 470 & 1 & 470 & $1.88 \mathrm{E}+03$ & 0.0147 & \\
\hline Residual & 0.250 & 1 & 0.250 & & & \\
\hline Cor total & $2.83 E+03$ & 4 & & & & \\
\hline
\end{tabular}

They were used to study the interaction effects of the independent factors on the responses at 1 time. The contour plot is formed by vertical axis and horizontal axis. The horizontal axis represents Soluplus and the vertical axis represents poloxamer.

\section{CONCLUSION}

In the present study, the SDs of the poorly soluble drug substance Ivacaftor were successfully prepared using HME. The in vitro dissolution test shows a significant increase in dissolution rate of SDs prepared by HME (95\%) in formulation FHM8 compared with plain Ivacaftor (9\%) within $30 \mathrm{~min}$. The release of drug was slightly on the higher side at initial time points from Ivacaftor SD technique by hot-melt extrusion technique. The increase in dissolution rate of Ivacaftor is in order of SDs of HME > plain drug substance. DOE results show the significant effect using Soluplus. The mechanism involved in solubilization by improved wetting of drug substance by hydrophilic carriers represents rich with microenvironment formed at the surface of the drug substance which leads to improves dissolution rate. The crystallinity nature of drug substance was reduced in SD technique by formulation with polymers. The results from FT-IR concluded that there was no defined interaction between Ivacaftor and carriers. DSC and XRD results showed a conversion of crystal structure toward to amorphous form of Ivacaftor. Finally concluded that HMT of Ivacaftor by using hydrophilic polymers would improved the aqueous dissolution rate, solubility and thereby enhance its systemic availability.

\section{AUTHORS' CONTRIBUTIONS}

The authors would like to thank Dr. Srinivas Lankalapalli for helping review, final corrections. and approved the final version of the manuscript.

\section{CONFLICTS OF INTEREST}

The authors declared that they have no conflicts of interest.

\section{REFERENCES}

1. Aitken-Nichol C, Zhang F, McGinity JW. Hot melt extrusion of acrylic films. Pharm Res 1996;13:804-8

2. Smita D, Sontakke SB. Solubility enhancement of gliclazide by solid dispersion method. Asian J Pharm Clin Res 2013;6 suppl 5:91-8.

3. Nidhalkhazaal M, Anastarik A, Ameeraabd R. Efficacy of combination solid dispersion technology on dissolution performance of nalidixic acid and cefdinir. Asian J Pharm Clin Res 2017;7:394-401.

4. Agrawal AM, Dudhedia MS, Zimny E. Hot melt extrusion: Development of an amorphous solid dispersion for an insoluble drug from mini-scale to clinical scale. AAPS Pharm Sci Tech 2016;17:133-47.

5. Wagh K, Crowley MS, Schroeder BC, Fredersdorf AS, Obara SH, Talarico MG. Design and evaluation of bilayer tablets of glimepiride and metformin hydrochloride with combination of hydrophilic and hydrophobic polymers by hot melt extrusion. Asian J Pharm Clin Res 2014; 7:300-4

6. Guns S, Dereymaker A, Kayaert P, Mathot V, Martens JA, Van den Mooter G, et al. Comparison between hot-melt extrusion and spraydrying for manufacturing solid dispersions of the graft copolymer of ethylene glycol and vinyl alcohol. Pharm Res 2011;28:673-82.

7. Homayouni A, Sadeghi F, Nokhodchi A, Varshosaz J, Afrasiabi Garekani H. Preparation and characterization of celecoxib dispersions in soluplus $(\mathbb{R})$ : Comparison of spray drying and conventional methods. Iran J Pharm Res 2015;14:35-50.

8. Huang S, O’Donnell KP, Delpon de Vaux SM, O’Brien J, Stutzman J, Williams RO $3^{\text {rd }}$, et al. Processing thermally labile drugs by hotmelt extrusion: The lesson with gliclazide. Eur J Pharm Biopharm 2017;119:56-67.

9. Hughey JR, Keen JM, Miller DA, Kolter K, Langley N, McGinity JW, et al. The use of inorganic salts to improve the dissolution characteristics of tablets containing soluplus ${ }_{\mathbb{Q}}$-based solid dispersions. Eur J Pharm Sci 2013;48:758-66.

10. De Meuter P, Rahier H, Van Mele B. The use of modulated temperature differential scanning calorimetry for the characterisation of food systems. Int J Pharm 1999;192:77-84.

11. Bunaciu AA, Udriștioiu EG, Aboul-Enein HY. X-ray diffraction: Instrumentation and applications. Crit Rev Anal Chem 2015;45:289-99.

12. Taylor LS, Zografi G. Spectroscopic characterization of interactions between PVP and indomethacin in amorphous molecular dispersions. Pharm Res 1997;14:1691-8.

13. Mehta S, Joseph NM, Feleke F, Palani S. Improving solubility of BCS class II drugs using solid dispersion. J Drug Deliv Ther 2014;4:7-13.

14. Zhang H, Yao M, Morrison RA, Chong S. Commonly used surfactant, tween 80, improves absorption of P-glycoprotein substrate, digoxin, in rats. Arch Pharm Res 2003;26:768-72.

15. Mohanty S, Jinno J, Kamada N, Miyake M, Yamada K, Mukai T. Dissolution enhancement of Seroquel by solid dispersion techniques. Asian J Pharm Clin Res 2016;11:284-7. 